\title{
ARVENSES ASOCIADAS A SISTEMAS DE PASTURAS EN LA ZONA BAJA DEL DEPARTAMENTO DEL QUINDÍO
}

\author{
WEEDS ASSOCIATED WITH PASTURE SYSTEMS IN THE LOW ZONE OF \\ THE DEPARTMENT OF QUINDIO
}

Libia Mayerly Cifuentes', María del Pilar Sepúlveda Nieto \& Germán Darío Gómez Marín².

${ }^{1}$ Programa de Biología, Centro de Estudios e Investigaciones en Biodiversidad y Biotecnología CIBUQ, Universidad el Quindío, Colombia.1mcifuentesg@uqvirtual.edu.com, msepulveda@uniquindio.edu.com

${ }^{2}$ Reserva El Ocaso, Centro de Estudios e Investigaciones en Biodiversidad y Biotecnología CIBUQ. Universidad del Quindío, germandario@uniquindio.edu.co.

\section{RESUMEN}

Se caracterizó la flora arvense asociada a sistemas de pasturas en el departamento del Quindío-Colombia ( $950 \mathrm{~m}$ a $1100 \mathrm{~m})$, también se identificaron los usos potenciales de la flora arvense asociada a este tipo de sistemas. Se registraron 80 especies, agrupadas en 70 géneros y 36 familias, las familias con mayor número de especies fueron Asteraceae con 13 especies, Leguminosae con 10, Cyperaceae con 7especies, Solanaceae con 5, Verbenaceae con 4 especies seguidas por Lamiaceae y Euphorbiaceae con 3 especies cada una.

En cuanto a los géneros con mayor número de especies en su orden son: Cyperus con 4 especies, Solanum con 3 especies, Solidago, Desmodium, Lantana, Hyptis y Euphorbia con 2 especies respectivamente. Dentro de los usos de las especies reportados por los pobladores de las fincas dedicadas a la ganadería en la zona cálida del Quindío se encontraron los siguientes: Apícola 37\%, Medicinal 16\%, Hospedera 14\%, fijadora de Nitrógeno N. $8 \%$, Toxica $6 \%$, Forraje 4\%, otros $15 \%$. Sin embargo, los propietarios de los predios dedicados a la ganadería en esta zona cálida del Quindío, tienen incluido dentro de sus prácticas tradicionales de manejo de los potreros la fumigación con herbicidas, ignorando las potencialidades de uso que tienen las arvenses que crecen asociadas a sus sistemas de pastura.

Palabras clave: Flora arvense, sistemas de pasturas, zona cálida y ganadería. 


\begin{abstract}
We characterized the weed flora associated with pasture systems in the department of Quindío-Colombia $(950 \mathrm{~m}$ to $1100 \mathrm{~m})$, we also identified potential uses of the weed flora associated with thise types of systems. There were reported 80 species, grouped in 70 genera and 36 families; the families with the greatest number of species were Asteraceae with 13 species, Leguminosae with 10, Cyperaceae,with 7especies, Solanaceae with 5, Verbenaceae with 4 species followed by Lamiaceae and Euphorbiaceae with 3 species each one.

As for the genera with the greatest number of species in their order are: Cyperus with 4 species, Solanum with 3 species, Solidago, Desmodium, Lantana, Hyptis and Euphorbia with 2 species respectively. Within the species uses reported by the residents of farms dedicated to cattle in the warm zone of Quindío, we found the following: Beekeeping 37\% Medical 16\%, Hospedería 14\%, nitrogen-fixing N. 8\% Toxica 6\%, Feed 4\%, others $15 \%$. However; the farms owners involved in livestock production in this hot area of Quindío, are included within their traditional pasture practices the management of weedkillers spraying, ignoring the potential use of weeds that grown together with their pasture systems.
\end{abstract}

Keywords: weed flora, pasture system, warm zone and livestock.

\section{INTRODUCCIÓN}

Desde sus orígenes la agricultura se ha ocupado de las "malezas o arvenses"; a pesar de ello no se ha logrado definir satisfactoriamente el término, aceptándose por lo general el concepto de que "maleza" es aquella planta que crece donde no se desea (1). Este concepto es inadecuado y obsoleto, ya que proviene ante todo de una visión sesgadamente antropocentrista, pues sin el hombre no habría malezas, ya que estas aparecieron a la par con la agricultura (2).

Según Pitty (1997) (3), todas las malezas tienen algunas ventajas y desventajas para el hombre. Algunas son de beneficio por que se usan en la medicina tradicional, son usadas como alimento para el hombre o sus animales, son apreciadas por sus flores vistosas y coloridas, sirven de alimento y refugio a los animales silvestres, reducen la erosión del suelo y aumentan el control natural de las plagas. Sin embargo, las mismas plantas u otras causan alergias, irritaciones y envenenamientos a los animales, son hospederas de plagas y reducen el valor de la tierra. Gómez \& Rivera 1987(4), consideran que del número total de especies (unas 350.000) de plantas conocidas, 30.000 se relacionan con el hombre, los animales y las plantas y unas 2.500 se consideran, "malezas" de importancia económica.

Por otro lado Matthei (1995) (5), estima que la radicación de las malezas es un proceso notablemente dinámico, en el cual la participación del hombre es incuestionable y en el que intervienen numerosos agentes destacándose entre ellos el hombre, agua, animales y el viento. Y el hombre es, sin lugar a dudas el principal agente de diseminación ya sea consciente o inconscientemente.

El departamento del Quindío, en la zona baja (950 $1400 \mathrm{~m}$ ), cuenta con un área aproximada de 19.659 ha. de tierras para fines ganaderos, lo que ha generado que la mayor cobertura del suelo haya sido sembrada en pastos y otros forrajes, de este modo el departamento se ha visto influenciado por muchas prácticas inadecuadas de manejo y cambios en el uso de la tierra, sumados al efecto de los factores ambientales que han modificado el paisaje original en los últimos años (6).

Según el Instituto Alexander Von Humboldt y la CRQ (2003) (7), la alta diversidad biológica en especies y ecosistemas en el departamento del Quindío, en evolución con los grupos humanos que los han transformado, se ve representada en los diferentes diseños y composición de los agroecosistemas del departamento. Aunque aún no se cuenta con un inventario y caracterización de los sistemas productivos locales a una buena escala, se pueden diferenciar un alto número de ellos. La tendencia en el manejo de estos agroecosistemas es a la homogenización, lo que tiene implicaciones negativas en términos de biodiversidad.

Con el propósito de caracterizar la flora arvense asociada a la zona de influencia de procesos ganaderos y el efecto del uso potencial de esta flora sobre dichos sistemas, se pretende presentar una alternativa que se considere importante y útil el reconocer la vegetación que acompaña las practicas ganaderas en la zona baja del 
departamento, en el sentido de brindar apoyo al sector ganadero para que conozca las ventajas de potencializar la flora arvense como plantas útiles, y sus beneficios a nivel medicinal, forrajero o como recuperadoras de suelo, así como las propiedades tóxicas que pueden representar un factor de riesgo para los animales.

En este sentido el objetivo del presente estudio consistió en caracterizar la flora arvense asociada a sistemas de pasturas en la zona baja de departamento del Quindío $(950 \mathrm{~m}$ a $1400 \mathrm{~m})$ y además identificar los usos potenciales de esta diversidad.

\section{MATERIALES Y MÉTODOS}

\section{Área de estudio.}

El área de estudio se ubicó en la zona occidental del departamento del Quindío, específicamente en aquellas fincas dedicadas a la ganadería extensiva en el rango altitudinal de 950 a 1400 metros, (municipios de Armenia, Calarcá, Buenavista, Génova, La Tebaida, Montenegro, Pijao y Quimbaya) incluye parte de la microcuenca del río La Vieja (figura 1).

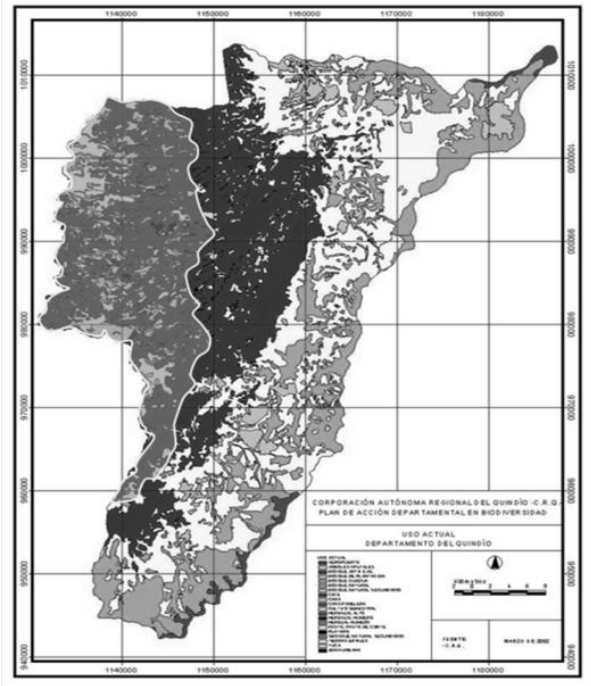

Figura 1. Zona de estudio, indicando el área de muestreo (zona cálida del departamento del Quindío)

\section{Fase de Campo}

El trabajo de campo se realizó en 16 estaciones de muestreo, distribuidas en fincas ganaderas con extensiones superiores a 20 hectáreas y ubicadas entre los 950 y los $1450 \mathrm{~m}$ de altitud.

En cada estación de muestreo se realizaron dos salidas de campo, dentro de las cuales se realizaron recorridos por toda la zona de potrero, ubicando la flora arvense asociada. De cada individuo se colectaron al menos dos muestras, dándole a cada una de ellas el mismo código para su posterior identificación.

Las colecciones fueron acompañadas de registros fotográficos y de una descripción general de las condiciones del sitio.

\section{Fase de Herbario}

La herborización de ejemplares se hizo siguiendo la metodología de Lot \& Chiang (1986) (8). Las colecciones de cada individuo incluyeron partes vegetativas y reproductivas, se prensaron en papel periódico y se llevaron al Herbario HUQ para su secado en estufa a 60 oC durante 2 a 3 días. Luego se hizo el respectivo montaje técnico de los ejemplares, incluyendo su etiqueta de campo, para proceder a su determinación taxonómica.

Los individuos ya procesados fueron identificados taxonómicamente de acuerdo a comparaciones con ejemplares de herbario determinados por especialistas en HUQ y COL, catálogos de plantas vasculares, libros especializados como las floras de Ecuador, Perú, Venezuela, Panamá, Costa Rica y Neotrópica, entre otras. Además, tratados de plantas vasculares de los siguientes autores Brako \& Zarucchi (1993) (9), Davidse, Sousa \& Knapp (1995) (10), Gentry, (1993) (11), Henderson, Galeano y Bernal (1995) (12), Moller \& León - Yánez (1999) y la base de datos del (14) Missouri Botanical Garden (www.mobot.org) (13). Las colecciones fueron depositadas en el Herbario Universidad del Quindío (H.U.Q.).

Usos potenciales de las arvenses asociadas a sistemas de pastura: Se diseñó una encuesta que fue aplicada en los sitios de muestreo, a los habitantes de la zona de estudio y en las plazas de mercado, considerando aspectos sobre el uso actual y potencial de las arvenses asociadas a los sitios de muestreo; de igual manera esta información fue complementada con una exhaustiva revisión bibliográfica.

\section{Índice de Valor de Uso (IVU)}

Se empleó el enfoque de consenso de informantes, metodología desarrollada por Adu-Tutu et al. (1979) (15), Phillips \& Gentry (1993) (16) y Phillips (1996) (17). Este valor expresa la importancia o valor cultural de una especie determinada para todos los informantes entrevistados. Para estimar el índice de valor de uso general de cada especie para todos los informantes (IVUs), se utilizará la fórmula: 


$$
\text { IVUs }=\frac{\sum_{\mathrm{i}} \mathrm{UVis}}{\mathbf{n}_{\mathrm{s}}}
$$

Donde: $\mathbf{U V i s}=$ número de usos mencionados por cada informante (i), para cada especie (s).

$\mathbf{n s}=$ número de informantes entrevistados .

Los datos de cada informante fueron usados para calcular el número promedio de usos de una especies en particular (18). De esta forma, los usos identificados por cada informante fueron promediados para obtener el índice de valor de uso general para cada especie. Esta estadística se puede aplicar a cualquier técnica de obtención de datos, en la que numerosas personas, proporcionan información sobre la gama de usos de alguna planta (19).

\section{Nivel de Uso Significativo TRAMIL (UST)}

Para estimar el nivel de uso significativo para cada especie y verificar su aceptación cultural, se utilizó la metodología propuesta por Germosén-Robineau (1995) (20). Esta metodología, expresa que aquellos usos medicinales que son citados con una frecuencia superior o igual al $20 \%$, por las personas encuestadas que usan plantas como primer recurso para un determinado problema de salud, pueden considerarse significativos desde el punto de vista de su aceptación cultural y, por lo tanto, merecen su evaluación y validación científica. El UST se calcula dividiendo el número de citaciones de uso para cada especie (s), entre el número de informantes encuestados, se propone la siguiente ecuación:

\section{UST $=\frac{\text { Uso Especie (s) }}{\text { nis }} \times 100$}

Donde: Uso Especie (s) = número de citaciones para cada especie.

nis $=$ número de informantes encuestados.

Las categorías de uso registradas se definen como sigue:

Apícola: Aquellas especies que presentan altas tasas de floración durante todo el año y que son visitadas por abejas y otro tipo de insectos melívoras.

Medicinal: Especies que se usan para el tratamiento de algún tipo de enfermedad o afección de la salud humana.

Hospedera: aquellas arvenses hospederas de insectos y demás artrópodos que tiene parte de su ciclo de vida en ellas, haciendo uso principalmente del follaje.

Fijadora de Nitrógeno (N): todas las especies principalmente de la familia Legunminosae que tienen la capacidad de fijar nitrógeno al suelo.

Toxica: todas aquellas especies que por la presencia de metabolítos en cualquiera de sus estructuras, representan algún grado de toxicidad para el ser humano y el ganado.

Forrajeo: especies cuyas hojas y tallos son consumidas por el ganado.

Otros: se refiere a otro tipo de usos poco frecuentes, asignados a las arvenses que crecen en las zonas de potreros; se agrupan las especies con usos mágico- religiosos, artesanales, ornamentales e incluso aquellas que no presentan un uso antrópico registrado.

\section{Fase de socialización}

Se diseñó una cartilla, dirigida al público interesado en plantas medicinales y aromáticas. En el material se incluyeron con el respectivo nombre científico, descripción morfológica, fotografías, usos y nombres comunes reportados en la literatura y por los encuestados.

Estrategia pedagógica: se elaboró una cartilla que presenta de manera clara y detallada los resultados obtenidos en esta investigación; adicionalmente la información obtenida sobre el número de especies y los potenciales usos de las arvenses que crecen asociadas a sistemas de pasturas se presentaron a modo de taller ante el gremio de ganaderos y público en general interesado en este tema en el departamento del Quindío.

\section{RESULTADOS}

\section{Diversidad de la flora arvense asociada a sistemas de pasturas:}

La diversidad florística de las arvenses asociadas a sistemas de pasturas en la zona cálida del departamento del Quindío, está representada por 80 especies, agrupadas en 70 géneros y 36 familias botánicas.

Las familias con mayor número de especies fueron Asteraceae con 13, Leguminosae con 10, Cyperaceae con 7 especies, Solanaceae con 5, Verbenaceae con 4 especies seguidas por Lamiaceae y Euphorbiaceae con 3 especies respectivamente. (Figura 2) (Anexo 1). 


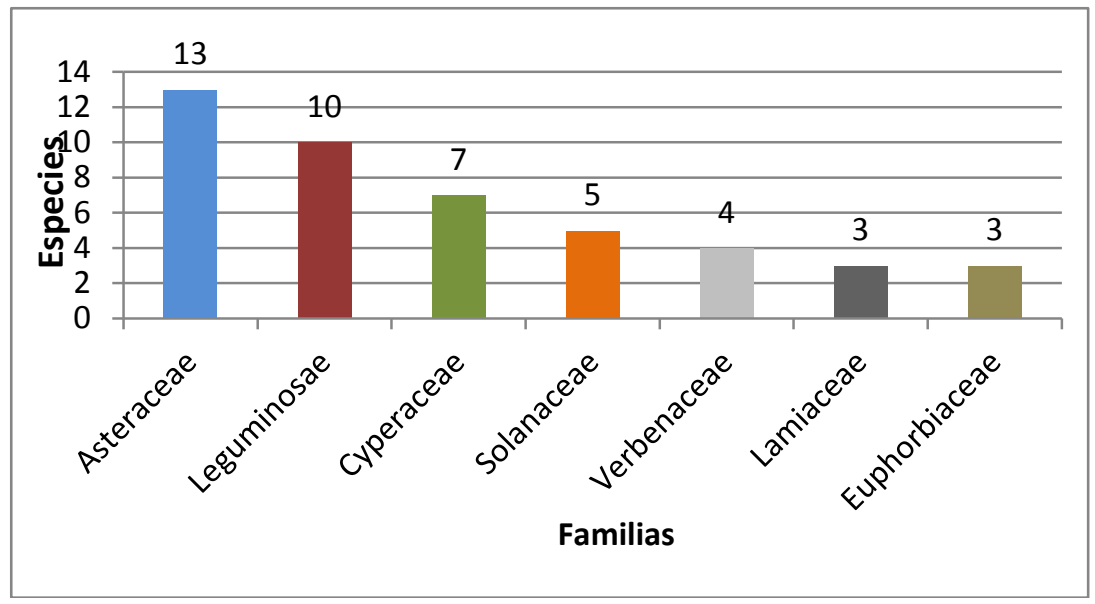

Figura 2. Familias que registraron el mayor número especies.

En cuanto a los géneros con mayor número de especies en su orden son: Cyperus con 4 especies las cuáles equivalen al $23 \%$, Solanum con 3 especies correspondiendo al 17\% del total de especies, Solidago, Desmodium, Lantana, Hyptis y Euphorbia con 2 especies respectivamente equivaliendo al $12 \%$ del total general de las especies reportadas. (Figura 3)

\begin{tabular}{|ll|}
\hline Cyperus & Solanum \\
Solidago & Desmodium \\
\hline
\end{tabular}

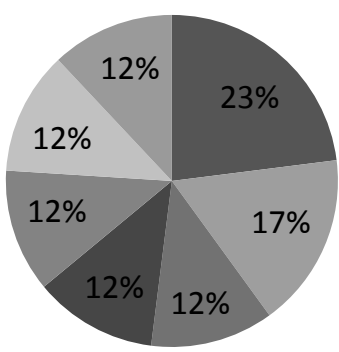

Figura 3. Géneros que registraron el mayor número especies.

Usos potenciales de la flora arvense asociada a procesos de ganadería: Del 100\% de las especies de arvenses registradas se reconoce por parte de los campesinos algún tipo de uso tradicional, sin embargo, las actividades de control y manejo de estas plantas -consideradas como malezas, por parte de los propietarios de los predios dedicados a la ganadería - están relacionadas con el uso de herbicidas como mecanismo de erradicación, conllevando a que los habitantes de estas fincas no hagan uso de estas especies, a pesar de conocer sus potenciales usos "no las usamos porque la mayoría de las veces están envenenadas".

\section{Índice de Valor de Uso (IVU)}

Teniendo en cuenta la encuesta realizada a 16 informantes sobre el uso de las especies de arvenses asociadas a los potreros de la zona cálida del Quindío, encontramos que las especies Desmodium intortum presentaron el mayor índice de valor de uso con 0,35 y Desmodium canum 0,32 seguidos por Austroeupatorium inulaefolium con 0.25 , Verbena litoralis 0.18 y Lantana cámara 0.15. (Tabla 1)

Tabla 1. Cinco especies con el mayor Índice de Valor de Uso (IVU)

\begin{tabular}{lc}
\hline \multicolumn{1}{c}{ ESPECIE } & IVU \\
\hline Desmodium intortum & 0,35 \\
Desmodium canum & 0,32 \\
Austroeupatorium & 0,25 \\
inulaefolium & \\
Verbena litoralis & 0,18 \\
Lantana camara & 0,15 \\
\hline
\end{tabular}

\section{Nivel de Uso Significativo TRAMIL (UST)}

Dentro de los usos de las especies reportados por los pobladores de las fincas dedicadas a la ganadería en la zona cálida del Quindío se encontraron los siguientes:

- Se reportaron 30 especies de uso apícola (37\%), 13 especies de uso medicinal (16\%), 11 especies que sirven como hospederas (14\%), 6 especies que son fijadoras de Nitrógeno (8\%), 3 especies utilizadas como forrraje para el ganado (4\%).

- Se reportan 5 especies $(6 \%)$ que representan peligro para la salud del ganado y las personas por su toxicidad.

- En otros usos se reportaron 12 especies que corresponden al $15 \%$ del total registrado. 
- El nivel de uso significativo (UST) reportado con una mayor frecuencia fue el apícola con el 37\%, seguido por el uso medicinal con 16\% y el de hospedera de insectos con un 15\%. (Figura 5)
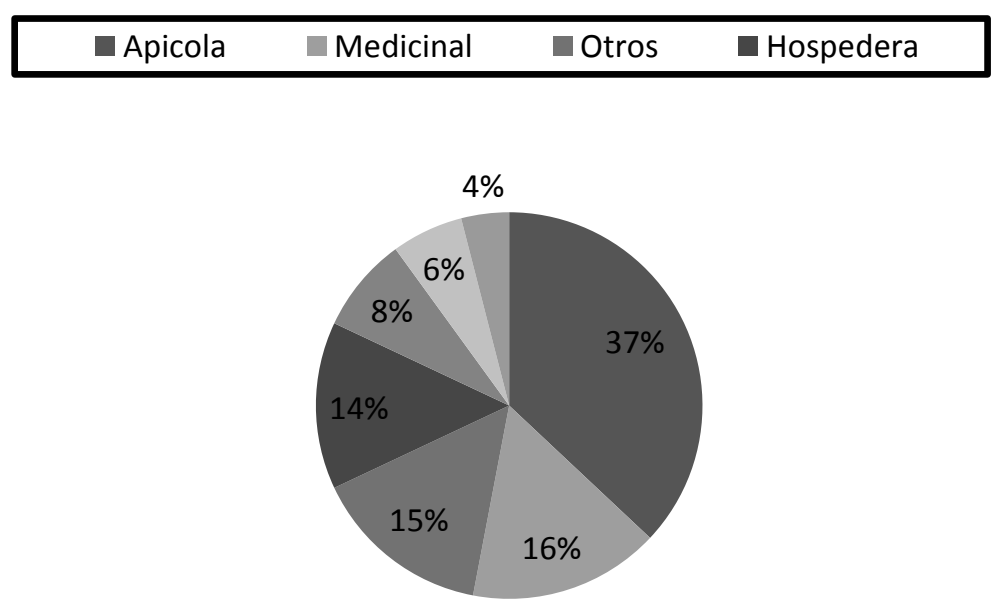

Figura 3. Tipos de usos registrados para las arvenses asociadas a pasturas en la zona cálida del departamento del Quindío.

Trabajo de extensión con los ganaderos:

Como estrategia para la difusión de la información obtenida en este trabajo se diseño un boletín informativo con la siguiente estructura: Introducción, definición de arvense, potenciales de uso de las arvenses, diagrama metodológico del proyecto, resultados del proyecto, nivel de uso significativo (UST), recomendaciones técnicas para el manejo de las arvenses, recomendaciones para el estudio de las arvenses.

Este boletín fue utilizado junto con una charla técnica para dar a conocer los resultados obtenidos ante la comunidad de ganaderos de la zona cálida del departamento. En total se realizaron tres charlas técnicas una por municipio (Quimbaya, Montenegro y La Tebaida).

De igual manera el proyecto fue socializado en un evento de carácter nacional (43 Congreso Nacional de Ciencias Biológicas en la ciudad de Yopal-Casanare), adicionalmente participamos durante dos emisiones de Meridiano Ambiental, espacio radial de la emisora de la Universidad del Quindío (UFM) en la realización de dos programas, en los cuales se abordo la temática central del proyecto "Arvenses asociadas sistemas de pasturas en la zona cálida del Quindío).

\section{DISCUSION}

Los resultados obtenidos en cuanto a la diversidad de especies de arvenses asociadas a los potreros de la zona cálida del Quindío permiten dar cuenta de la alta diversidad florística que puede estar asociada a un mo- nocultivo como el ganadero; de manera especifica estudios relacionados con arvenses asociadas a otro tipo de cultivos en Colombia como: café (Vélez et al. 1998 quienes reportan 95 especies) (21), banano (Pinilla $G$ \& García C 2002 encontraron 108 especies asociadas a este cultivo) (22) y palma de aceite (Rodríguez et al. 1998 mencionan 65 especies) (23) estas cifras indican que este tipo de monocultivos al igual que los potreros de la zona cálida del Quindío posee una diversidad alta en términos de especies de arvenses.

La alta diversidad de arvenses que crecen asociadas a los sistemas ganaderos obedece en gran medida a la capacidad reproductiva y a la alta tolerancia de estas especies a condiciones ambientales extremas Rodríguez et al. 1998 proponen que las arvenses de cultivo permanecen gracias a adaptaciones tales como: bajo consumo de agua, crecimiento rastrero o de porte inferior a 20 $\mathrm{cm}$, sistema radical ralo y superficial, buena capacidad de cubrimiento y dominancia poblacional y una alta producción de semillas y propágalos vegetativos.

Igualmente Murgueitio et al. 2003 (24), mencionan que las arvenses asociadas a sistemas productivos ganaderos son consideradas como las más "agresivas" y mejor adaptadas a las condiciones que posee un potrero, algunas de las adaptaciones mas importantes son las siguientes: rápido crecimiento vegetativo, sistemas radiculares superficiales, ciclos de vida cortos, alta producción de semillas, dispersión de corta y larga distancia, longevidad y latencia de las semillas, capacidad de germinar bajo diferentes ambientes 
De manera específica en este trabajo encontramos ejemplos particulares sobre la alta capacidad de adaptación de estas arvenses que crecen asociadas a potreros, especies como: Hydrocotile umbellata y Dichromena ciliata se observaron creciendo tanto en áreas inundadas temporalmente y en zonas secas y se observó además que el ganado actúa como dispersor de gran parte de ellas cuando las consumen y transportan los frutos pagados a su pelaje.

Respecto al manejo de la arvenses asociadas a los potreros de la zona cálida del Quindío es necesario considerar la premisa básica propuesta por Smith (1990) (25) para cualquier programa de manejo integrado de arvenses las medidas de control deben ser aplicadas sólo después que las arvenses alcancen el umbral que causa pérdidas económicas al cultivo.

Según Coble \& Mortensen (1992) (26) el principio de estimar la población de arvenses antes de realizar alguna acción de manejo es una premisa fundamental para cualquier programa de Manejo Integrado de Arvenses. El manejo de arvenses ha venido cambiando desde la aplicación general de herbicidas químicos, tratamientos mecánicos y culturales, por manejo de arvenses basado en la solución de situaciones específicas y puntuales.

En general no es óptimo el control de todas las arvenses y la decisión de no controlarlas debe ser tomada sí la utilidad adicional asociada con el rendimiento más alto es menor que el costo del control (27).

Según Zimdahl (1980) (28) son pocas las arvenses que usualmente no afectan el rendimiento de los cultivos, sin embargo existen densidades de población de arvenses que pueden ser toleradas por los cultivos sin disminuir significativamente los rendimientos.

Si consideramos los costos ambientales que genera el control profiláctico de las arvenses (erradicación con herbicidas) Rodríguez et al. 1998 plantean que se debe recordar que el control químico no es el único y de ninguna manera el más efectivo, pero se recomienda como complemento del control cultural, mecánico y manual, haciendo uso de la combinación de ellos, de acuerdo con la situación que se presente.

Rodríguez et al. 1998 reconocen que un alto porcentaje de fincas en donde se hace el control químico de arvenses presentan prácticas deficientes tal como lo evidenciamos en esta investigación, algunas de ellas son: las aplicaciones son de carácter generalizado, muy pocas veces se hace parcheo, las dosis de herbicidas no están estandarizadas, el ciclo calendario siempre se hace con un solo herbicida (Independiente de las malezas existentes en cada uno de los lotes), la selección del herbicida generalmente esta a potestad del productor o administrador, el agua para la preparación de la mezcla herbicida es sacada de aguas estancadas, quebradas caños, ríos, tanques de beneficio, etc, se aplica en condiciones ambientales adversas, los residuos de los herbicidas son dispuestos irregularmente (recipientes y bombas), no se guardan las mínimas normas de seguridad (protección personal).

Respecto a las implicaciones del control químico de arvenses durante este trabajo pudimos evidenciar de manera generalizada que cuando esta práctica es implementada por los propietarios de los predios, no se tiene una estrategia clara frente al manejo de los riesgos que acarrean el manejo de sustancias toxicas de alta peligrosidad como lo son los herbicidas.

Para considerar la pertinencia del control de las arvenses asociadas a cultivos Chee et al. (1992) (29), sugieren que es necesario clasificar las arvenses de acuerdo a su agresividad el fin de realizar un manejo eficiente y selectivo; los mismos autores proponen un ejemplo de clasificación de las arvenses: Clase A: plantas benéficas que deben ser explotadas para suprimir arvenses agresivas, conservar el suelo contra la erosión, y disminuir los costos de las desyerbas. Clase B: Plantas aceptables en la plantación, pero requieren ser manejadas cuando sea necesario. Clase $\boldsymbol{C}$ : Plantas que interfieren en alto grado con los cultivos.

Estos mismos autores plantean una serie de características que debe tenerse en cuenta para considerar una arvense como benéfica o noble: tolerantes al pisoteo, tolerantes a la sequía y a las condiciones de encharcamiento, alta producción de estructuras florales (alimento para fauna benéfica), facilidad de propagación sexual y vegetativa.

El hecho de facilitar el establecimiento de arvenses "nobles", permite disminuir los costos de las desyerbas entre 85 y $95 \%$ y proteger los suelos contra la erosión con una eficiencia entre el 95 y 97\% (31). 


\section{CONCLUSIONES}

1. Existe alta diversidad de géneros y especies arvenses asociadas a los sistemas de pasturas de la zona baja del Quindío, pese a la utilización del modelo tradicional de explotación ganadera que prevalece en la región.

2. Dentro de los usos de las especies reportados por los pobladores de las fincas dedicadas a la ganadería en la zona cálida del Quindío se encontraron los siguientes: Apícola 37\%, Medicinal $16 \%$, Hospedera 14\%, fijadora de Nitrógeno N. $8 \%$, Toxica $6 \%$, Forraje $4 \%$, otros $15 \%$.

3. Las arvenses Asclepia curassavica, Mormodica charantia, Lantana camara y Renealmia cernua son especies con altos contenidos de metabolítos secundarios tóxicos que pueden llegar a ser mortales en el caso de la lantanina, y abortivos como el contenido de las flores de Renealmia cernua.

4. Los propietarios de los predios dedicados a la ganadería en esta zona cálida del Quindío, tienen incluido dentro de sus prácticas tradicionales de manejo de los potreros la fumigación con herbicidas, desconociendo las potencialidades de uso que tienen las arvenses que crecen asociadas a sus sistemas de pastura.

5. Este tipo de trabajo aporta información útil sobre las arvenses que se pueden encontrar asociadas a los procesos de ganadería en el departamento del Quindío y de manera particular en la zona cálida y por lo tanto los resultados obtenidos pueden ser utilizados para la generación de estrategias de manejo de estas arvenses con el objeto de reducir los costos en que se incurre actualmente al utilizar herbicidas para su regulación.

6. El gremio ganadero en el Quindío no posee un programa para el manejo integrado de arvenses asociadas a sus sistemas ganaderos que permita hacer una intervención mínima con el uso de herbicidas.

\section{RECOMENDACIONES}

En los sistemas tradicionales de ganadería de la zona cálida del Quindío pudimos evidenciar un sistema de manejo que busca bajo mecanismos de control principalmente de tipo químico mantener los potreros libres de las llamadas "malezas ".

Por lo tanto recomendamos dos estrategias para el manejo de arvenses, que permiten de una forma económica y armonizada con el ambiente controlar este tipo de hierbas que se asocian de manera permanente a los potreros.

Erradicación manual: Es la eliminación total de arvenses en el campo, de manera manual con la ayuda de herramientas tales como machetes, azadones y palines convirtiéndose en una práctica amigable con el ambiente y que genera beneficios a corto y largo plazo si se hace con cierta regularidad (lo recomendado es cada tres meses) y de manera permanente.

Remedial o de contención: Esta estrategia es usada para mantener la población de arvenses en un nivel específico bajo, tolerando la presencia de alguna población de arvenses en el cultivo, siempre y cuando sean especies que no representen pérdidas económicas y no afecten los rendimientos de sistema. Las prácticas remédiales son de gran valor potencial por presentar el menor costo y ser ambientalmente sanas.

Para poner en práctica esta estrategia es fundamental estimar la población y el tipo de arvenses antes de tomar alguna acción remedial o de contención que puede ser en la mayoría de las veces la erradicación manual selectiva de las arvenses presentes en el cultivo.

Este tipo de práctica de control da la posibilidad de que se pueda hacer uso de muchas especies de arvenses desde diferentes campos (medicinal, apícola, fijación de $\mathrm{N}$. entre otros).

Es indiscutible que para algunos casos particulares los propietarios de predios ganaderos para reducir los costos en la mano de obra utilicen prácticas como la Profilaxis, la cual incluye la aplicación de herbicidas, este manejo puede resultar en detrimento de la calidad ambiental, desprotección total de los suelos, además de desperdicio de herbicida y dinero.

Respecto a esta práctica de manejo encontramos que 
en la mayoría de las veces el proceso no se hace con las especificaciones técnicas que se requieren para reducir el riesgo que implica la manipulación de los herbicidas tanto para los que hacen la aplicación como para el ambiente en general y de manera específica para los cuerpos de agua próximos a estos potreros

\section{AGRADECIMIENTOS}

Los autores de este trabajo agradecemos a la Vicerrectoría de Investigaciones de la Universidad del Quindío por la financiación, al CIBUQ por el apoyo logístico y a todos los ganaderos de la zona cálida del departamento del Quindío por la colaboración y el apoyo brindado durante el desarrollo de este.

Anexo 1. Listado de las familias y especies de arvenses asociadas a los potreros de la zona cálida del Quindío.

\begin{tabular}{|c|c|c|c|}
\hline FAMILIA & ESPECIE & FAMILIA & ESPECIE \\
\hline Acanthaceae & Thumbergia alata & Leguminosae & Senna hisurta \\
\hline Amaranthaceae & Cyathula postrata & Leguminosae & Desmodium canum \\
\hline Apiaceae & Hydrocotile umbellata & Leguminosae & Teramus voluvilis \\
\hline Apiacee & Spananthe paniculata Jacq & Leguminosae & Desmodium Intortum \\
\hline Asclepidaceae & Asclepia curassavica & Leguminosae & Zornia diphylla \\
\hline Asteraceae & Austroeupatorium inulaefolium & Leguminosae & Stilosanthes guianensis \\
\hline Asteraceae & Emilia coccinea & Leguminosae & Chamaecrita nictitans \\
\hline Asteraceae & Mikania scandens & Leguminosae & Dioclea sp. \\
\hline Asteraceae & Ageratum conyzoides & Lytraceae & Cuphea racemosa \\
\hline Asteraceae & Fleischamia microstemos & Malvaceae & Sida rhombifolia \\
\hline Asteraceae & Pseudoelephantopus spicatus & Melastomataceae & Tibouchina cf longifolia \\
\hline Asteraceae & Melanthara nivea & Miosaceae & Mimosa pudica \\
\hline Asteraceae & Synedrella nodiflora & Onagraceae & Jussiaea suffructicosa \\
\hline Asteraceae & Bidens pilosa $L$. & Onagraceae & Ludwigia peruviana \\
\hline Asteraceae & Centratherum puntctatum & Piperaceae & Piper aduncun \\
\hline & Cassini & Poaceae & Paspalum paniculatum \\
\hline Asteraceae & Jaegeria hirta (Lag) Less & Poaceae & Cynodon dactylon \\
\hline Asteraceae & Solidago oleraceaus & Polygonaceae & Polygonum segetum Kunth \\
\hline Asteraceae & Crepis japonica (L.) Benth & Ponteridaceae & Heteranthera reniformis \\
\hline Asteraceae & Solidago chilesis Meyen & Portulacaceae & Portulaca oleracea \\
\hline Boraginaceae & Cordia polycephala & Rubiaceae & Borreria alata \\
\hline Campanulaceae & $\begin{array}{l}\text { Hippobroma longiflora (L.) G. } \\
\text { Don }\end{array}$ & Sapindaceae & $\begin{array}{l}\text { Cardiospermum halicacabum } \\
\text { L. }\end{array}$ \\
\hline Caryophyllaceae & Drymaria cordata (L.) Willd ex & Solanaceae & Solanum hirtum \\
\hline Commelinaceae & Commelina diffusa & Solanaceae & Cestrum microcalyx \\
\hline Cucurbitaceae & Mormodica charantia & Solanaceae & Browlia americana $L$. \\
\hline $\begin{array}{l}\text { Cucurbitaceae } \\
\text { Cvperaceae }\end{array}$ & Melontria pendula & Tiliaceae & Triumpheta bogotensis \\
\hline $\begin{array}{l}\text { Cyperaceae } \\
\text { Cyperaceae }\end{array}$ & $\begin{array}{l}\text { Cyperus flavus } \\
\text { Scleria pterata }\end{array}$ & Urticaceae & Phenax spanerati \\
\hline Cyperaceae & Dichromena ciliata & saceae & Pilea hyalina Fenzl, Nov. \\
\hline Cyperaceae & Eleocharis elegans & Verbenaceae & Stachvtarnheta cavennensis \\
\hline Euphorbiaceae & Euphorbia hirta & Verbenaceae & Lantana camara \\
\hline Euphorbiaceae & Phillantus urinaria & Verbenaceae & Verbena litoralis \\
\hline Euphorbiaceae & Euphorbia heteropylla & Vitaceae & Cissus cucumis Standl Field. \\
\hline Heliconiaceae & Heliconia episcopalis & & Mus \\
\hline Lamiaceae & Hyptis capitata & Zingiberaceae & Renealmia cernua \\
\hline $\begin{array}{l}\text { Lamiaceae } \\
\text { Lequminosae }\end{array}$ & $\begin{array}{l}\text { Stachys micheliana } \\
\text { Centrosema nuhescens }\end{array}$ & Zingiberaceae & Hedichium coronarium \\
\hline
\end{tabular}




\section{BIBLIOGRAFÍA}

1. Bowen, Calvache, H.; Nel, P. \& Aldana et al., (citados por Vélez et al. 1998). Flora of the Venezuelan Guayana, 5. 1980.

2. Fuentes, C, Gomez \& Clavijo, J. Memorias conferencia: Manejo de malezas en plantaciones de banano y plátano. INAGRU. Apartadó Antioquia-Colombia.. 1998, pp 21.

3. Pitty, A (ed.). Introducción a la Biología, ecología y manejo de malezas. Zamorano Academic Press. Honduras. Plagas (Costa Rica) 1997, 20/21:1-7.

4. Gómez \& Rivera. Plantas medicinales y venenosas de Viterbo, Caldas, descripción y usos. Colegio Nazario Restrepo. 1994.

5. Matthei. Plant Systematics: A Phylogenetic Approach., second Edition. 2002.

6. Rivera, Ecuador-Manejo integrado de plagas en banano y plátano. Banana Export. Año 2, edición No. 5 Septiembre-Octubre 1999. CONABAN, Guayaquil- Ecuador. 16-18.

7. Instituto Alexander Von Humboldt y CRQ. Conocimiento, conservación y uso sustentable de la diversidad biológica"Propuesta técnica para el desarrollo del programa de Biodiversidad para el Quindío 2003-2012”. OPTIGRAF. 2003.

8. Lot a. y Chiang F. (Compiladores). Manual de Herbario. Consejo Nacional de la Flora de México. 1986, 142 p.

9. Brako \& Zarucchi. Especies Vegetales Promisorias de los Países del Convenio Andrés Bello. Tomos I-X11. Editorial Guadalupe Ltda. Bogotá. 1993.

10. Davidse, Sousa \& Knapp. Control de plagas de plantas y animales. Ediciones ciencia y técnica S.A. Naucalpan, Edo de México. 1988. 25-169. Oil Palm Papers (Costa Rica) 1995,19:1-22.

11. Gentry, Flora del estado Monagas - Malezas. 1993.

12. Henderson, Galeano \& Bernal. Entomofauna benéfica de las plantaciones de palma, en América tropical. Palmas, 1995.

13. Moller \& León - Yánez. Plant species attractive to beneficial Meyrick, en palma de aceite. Palmas (Colombia) 1999, 18(1):11-20

14. Missouri Botanical Garden (www.mobot.org).

15. Adu-Tutu, M; Afful, Y; Asante-Appiah, K; Leberman, D; Hall, J; Lvinlewis, M. 1979. Chewing Stick Usage in Southern Ghana. Econ Bot. 1979, 33(3):320-328.

16. Phillips, O \& A, Gentry. The Useful Plants of Tambopata, Perú: I. Statistical Hypotheses Test with a New Quantitative Technique. Econ Bot. 1993, 47(1):15-32.

17. Phillips, O. Some Quantitative Methods for Analyzing Ethnobotanical Knowledge, The New York Botanical Garden. 1996.

18. Cotton, C. Ethnobotany. Principles and Aplications, $2^{\mathrm{a}}$ ed. Baffins Lane, Chichester, West Sussex; John Wiley \& Sons. 1999.

19. Martin GJ. Ethnobotany. A methods Manual. London: Chapman \& Hall. 1995.

20. Germosén-Robineau, L editor. Hacia una farmacopea vegetal caribeña. Edición TRAMIL 7. Enda - Caribe, UAG \& Universidad de Antioquia. Santo Domingo. 1995.

21. Vélez, M., Agudelo, C. Macias, D., Flora Arvense de la región Cafetera Centro- Andina de Colombia. Tomo I. Litografía López Editores. Armenia. 186p. 1998.

22. Pinilla G \& García C. Manejo integrado de arvenses en plantaciones de banano (Musa AAA) 2002* Comercializadora Internacional Uniban S.A. C.I UNIBAN S.A. Dirección Agricultura. Apartadó, Antioquia, Colombia. 2002.

23. Rodríguez G, Fariñas, Díaz R, Silva-Acuña \& Elda. Reconocimiento de plantas arvenses en plantaciones de palma aceitera, 1998.

24. Murgueitio E., Ibrahim M., Ramírez E., Zapata A., Mejía C. \& Casasola F. 2003 Usos de la tierra en fincas ganaderas: Guía para el pago de servicios ambientales en el proyecto Enfoques silvopastoriles integrados para el manejo de ecosistemas. CIPAV, CATIE \& UCA-NITAPLAN. Cali Colombia.

25. Coble \& Mortensen. Arachis pintoi como alternativa sostenible en el manejo integrado de malezas en vid. Agronomía colombiana. 1999. 16 (1-3), 51-59.

26. King Campbell. C., Kellogg. E., Stevens. P., Donoghue. M. Malezas ecofisiología y estrategias de control. Facultad de Agronomía. Pontificia Universidad de Chile. 402 pp. 1998. 
27. Chee N. Chase, M Delvare, G. 1992 Monocot Relationships: An Overview. Am. J. Bot, 1992. 91 (10): 1645 1655.

27. Rivera, Plantas medicinales en los Andes de Bolivia. Botánica Económica de los Andes Centrales Editores: M. Moraes R., B. Øllgaard, L. P. Kvist, F. Borchsenius \& H. Balslev Universidad Mayor de San Andrés, La Paz. 1997, 268-284. 